\title{
Enhancement of MRI Prostate Image Using Histogram Equalization Techniques
}

\author{
N.Gopinath, A.Chinnasamy, M.SureshAnand, V. Pandimurugan
}

\begin{abstract}
In this cutting edge world, Medical image processing in computerized field needs a compelling MRI image modality with less commotion and improved contrast of image. This is conceivable by utilizing image enhancement methodology. Image enhancement is referenced as a system of changing or altering image so as to make it progressively sensible for explicit applications and is utilized to enhance or improve contrast proportion, splendor of image, expel clamor from image and make it less hard to perceive. The purpose behind inclining toward Medical Resonance Imaging (MRI) is that it is a mind boggling medical technology which gives more useful information regarding malignancy, stroke and various another ailments. It helps the doctors to distinguish the diseases more adequately. MRI has exceptionally low difference proportion. To improve the contrast of MRI image, we utilized Histogram equalization technique. In which, Histogram Equalization, Local Histogram Equalization, Adaptive Histogram Equalization and Contrast Limited Adaptive Histogram Equalization techniques were used and it is pondered.
\end{abstract}

Index Terms:: Medical image processing, MRI Prostate image, Image enhancement, Histogram Equalization, Local Histogram Equalization, Adaptive Histogram Equalization and Contrast Limited Adaptive Histogram

\section{INTRODUCTION}

Medical Image processing has assumed greater significance in recent years because of better anticipation and analysis abilities that have accompanied progressions in it. It plays a very important role in the finding and forecast of malignant growth based ailments created in human body most generally in lungs, cerebrum, bosom and prostate organ. Medical imaging modalities have been developing to capture high quality medical images [1]. Magnetic Resonance Imaging (MRI) is one of the common and widely used image modality in Medical image processing.

Magnetic Resonance Imaging is a tomographic imaging technique that uses a strong magnetic field and radio waves to produces images of internal physical and chemical characteristics of an object from externally measured nuclear magnetic resonance (NMR) signals [2]. MR images are incredibly wealthy in data content. It is employed in medical field to generate very good quality images of the human body parts.

Revised Manuscript Received on October 12, 2018.

N.Gopinath, Department of Computer Science Engineering Sri Sairam Engineering College, Chennai.

A. Chinnasamy, Department of Computer Science Engineering, Sri Sairam Engineering College, Chennai.

M. SureshAnand, Department of Computer Science Engineering, Sri Sairam Engineering College, Chennai.

V. Pandimurugan, Department of Information Technology, Hindustan Institute of Technology, Chennai
The MRI is utilized for many purposes such as diagnosing brain, prostate tumours, multiple diseases, and spinal infections; to visualize shoulder injuries, tumours in bones and strokes [3]. The MRI images clearly shows the tissues that are harmed or infected and these images are stored on a $\mathrm{PC}$ for processing and further references. To give appropriate analysis and great outcomes, doctors are given the various consequences of enhanced images. Enhancement is a crucial undertaking in digital image processing and examination, planning to improve the appearance of image as far as human splendour recognition.

Image enhancement, which is one of the most significant technique in digital image processing, plays a vital role in numerous fields like medicinal image handling, remote sensing, speech recognition, microscopic imaging and also in many other image and video processing applications. This Image enhancement process comprises of a collection of techniques that are utilized to improve the visual appearance of an image. It is a procedure by which the visual quality and the overall appearance of an image are improved in order to separate the spatial features of the image [4]. The main objective of Image enhancement is to improve the perception of data contained in the image for human viewers. It creates output image that abstractly looks better than the original image by changing the pixel's intensity of the input image. A very well-liked performance for contrast upgrade is Histogram Equalization (HE).

A histogram is the estimation of the likelihood dispersion of a specific kind of information. An image histogram is a sort of histogram which offers a graphical portrayal of the tonal dispersion of the gray values in a digital image [5]. Image histograms are one of the important tools for inspecting images. The $\mathrm{X}$ axis of the graph speaks to the tonal varieties, while the $\mathrm{Y}$ axis speaks to the quantity of pixels in that specific tone. The left half of the $\mathrm{X}$ axis speaks to the dark and dim territories, the center speaks to medium dim and the right hand side speaks to light and unadulterated white regions. The $\mathrm{Y}$ axis represents the size of the area that is captured in each one of these zones [6].

\section{PROPOSED METHOD}

In order to improve the contrast of MRI images various image enhancement methods related to histogram techniques were proposed. They are Histogram Equalization (HE), Local Histogram Equalization (LHE), Adaptive Histogram Equalization (AHE) and Contrast Limited Adaptive Histogram Equalization (CLAHE) techniques. These techniques are compared with some of the comparison parameters like Chi-square, Intersection, E-Canberra, 


\section{Enhancement of MRI Prostate Image Using Histogram Equalization Techniques}

Euclidean, Dnon-IS, Square-Chord to show its efficiency.

\section{A. Histogram Equalization:}

Histogram equalization ( $\mathrm{HE}$ ) is a widely used technique for contrast enhancement since it is easy to utilize and better in execution on a wide range of images. It is most usually utilized in the areas like medical image processing, radar signal processing and so forth. HE works by smoothing the histogram of input image and stretches dynamic scope of gray levels by using cumulative density function (CDF) of the image [7]. Histogram of an image represents the relative frequency of occurrence of gray levels to preserve mean splendor of the input image. The HE method re-maps the gray levels of input image by reassigning intensity values of pixels to make a uniform intensity distribution. The histogram equalization algorithm might be partitioned into two sorts: local histogram equalization and global histogram equalization. The local histogram equalization well upgrade nearby subtleties of the image and it might be isolated into three sorts: overlapping sub-block, nonoverlapping sub block, and partially overlapping sub-block. The nonoverlapping sub-block method is all around once in a while utilized for its conspicuous square impacts; the overlapping sub-block method is also not used in practice for its large amount of calculation and low processing speed; the partially overlapping sub-block method can speed the computation, yet it is moderately perplexing [8]. Compared to the local histogram equalization algorithm, the global algorithm has certain favourable circumstances in handling speed, but has disadvantages in enhancing effects.

\section{B. Local Histogram Equalization:}

Local histogram equalization (LHE) $[9,10]$ utilizes a sliding window wherein, for every pixel, local histograms are computed from the windowed neighbourhood to deliver a local grey level remapping for each pixel. The grey level of the pixel at the middle of the neighbourhood is changed according to the local grey level remapping for that pixel. LHE is fit for great contrast enhancement which can now and again be considered over-enhancement. LHE-based methods are generally requiring more computation than other methods in light of the fact that local histogram should be fabricated and handled for every image pixel.

\section{Adaptive Histogram Equalization:}

This is an expansion to conventional Histogram Equalization method. It enhances the contrast of images by changing the qualities in the Intensity image. AHE works on small data regions (tile's) as opposed to the entire image. Each tile's contrast is upgraded, with the goal that the histogram of the output region matches the predetermined histogram [11]. The neighbouring tiles are then joined utilizing bilinear interpolation so as to dispose artificially induced boundaries. The contrast, particularly in homogeneous areas, can be restricted so as to abstain from intensifying the noise which may be available in the present image.

\section{Contrast Limited Adaptive Histogram Equalization:}

Contrast limited adaptive equalization is a modified method of adaptive histogram equalization. It is one of the spatial domain methods of contrast improvement. It was originally produced for successfully enhancing the low contrast medical images. This technique segregates the images into related regions and finds the equalization to each and every region [12]. It flattens the distribution of grey levels and in this way makes concealed highlights of the image progressively obvious. It produces the optimal equalization as far as most extreme entropy and furthermore confines the contrast of an image. This technique is very useful where the brightness requirement is high.

The pros and cons of the above said four techniques were given in short:

\begin{tabular}{|c|c|c|}
\hline $\begin{array}{l}\text { Name of the } \\
\text { Technique }\end{array}$ & pros & cons \\
\hline $\begin{array}{c}\text { Histogram } \\
\text { Equalization (HE) }\end{array}$ & $\begin{array}{l}\text { Enhance } \\
\text { contrast }\end{array}$ & $\begin{array}{l}\text { Over contrast } \\
\text { enhancement }\end{array}$ \\
\hline $\begin{array}{l}\quad \text { Local } \\
\text { Histogram } \\
\text { Equalization } \\
\text { (LHE) } \\
\end{array}$ & $\begin{array}{l}\quad \text { Offers an } \\
\text { excellent } \\
\text { enhancement of } \\
\text { image contrast }\end{array}$ & $\begin{array}{l}\text { Computationally } \\
\text { very slow, requires } \\
\text { high number of } \\
\text { operations per pixel. }\end{array}$ \\
\hline $\begin{array}{l}\quad \text { Adaptive } \\
\text { Histogram } \\
\text { Equalization } \\
\text { (AHE) }\end{array}$ & $\begin{array}{l}\text { Better than } \\
\mathrm{HE}\end{array}$ & $\begin{array}{l}\text { Enhance noise } \\
\text { and observation of } \\
\text { details becomes } \\
\text { difficult }\end{array}$ \\
\hline $\begin{array}{l}\quad \text { Contrast Limit } \\
\text { Adaptive } \\
\text { Histogram } \\
\text { Equalization } \\
\text { (CLAHE) }\end{array}$ & $\begin{array}{l}\text { Removes } \\
\text { draw backs of } \\
\text { AHE }\end{array}$ & $\begin{array}{l}\text { Brightness loss in } \\
\text { the processed image }\end{array}$ \\
\hline
\end{tabular}

\section{PROPOSED FLOWCHART}

The flow chart for the proposed method is given below:

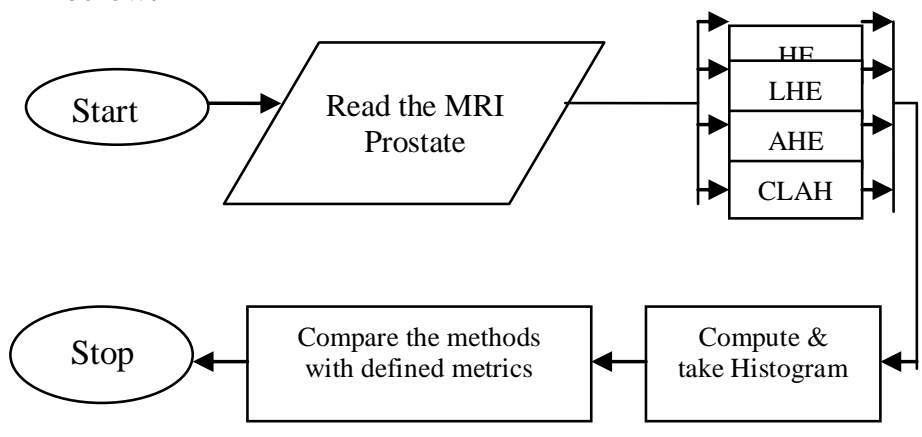

Fig 1 shows the flow chart of proposed method

\section{PROPOSED ALGORITHM}

The general methodology to enhance an image using Histogram equalization is depicted. The following are the steps to implement the algorithm:

Step 1: Read the MRI prostate image.

Step 2: Convert the MRI prostate image into Gray level MRI image.

Step 3: To enhance the contrast, perform the Histogram Equalization (HE) procedure on the MRI prostate input image.

Step 4: Take the histogram of the enhanced image.

Step 5: Perform the Local Histogram Equalization (LHE) technique on the input MRI Prostate image to enhance the contrast. 
Step 6: Repeat step 4

Step 7: Perform the Adaptive Histogram Equalization (AHE) technique on the input MRI Prostate image to enhance the contrast.

Step 8: Repeat step 4

Step 9: Perform the Contrast Limit Adaptive Histogram Equalization (CLAHE) technique on the input MRI prostate image to enhance the contrast.

Step 10: Compare the enhanced images of HE, LHE, AHE and CLAHE techniques with the help of defined metrics to show the efficiency of those techniques.

\section{RESULTS AND DISCUSSIONS}

The following are the resultant pictures of the proposed MRI Prostate image enhancement utilizing various Histogram Equalization techniques:

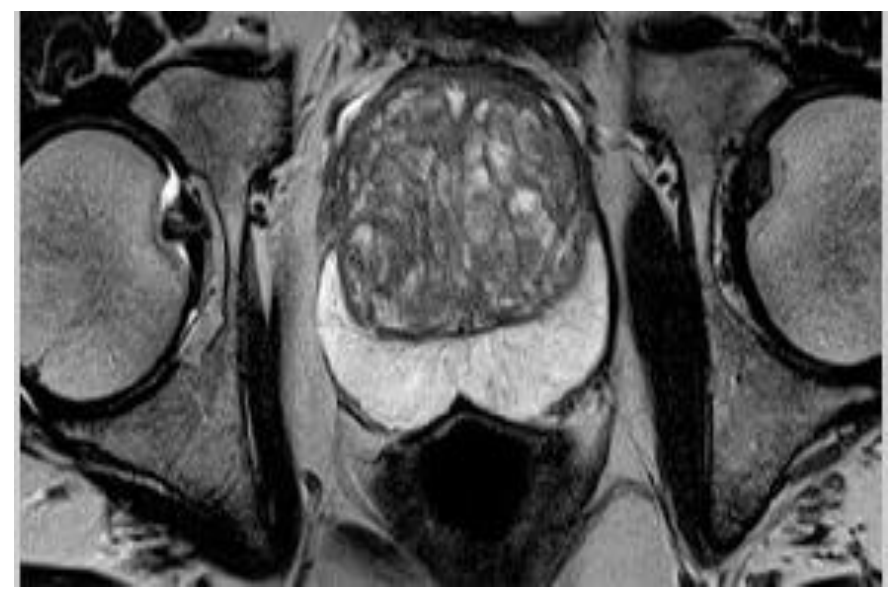

Fig 2. Original MRI Prostate Image

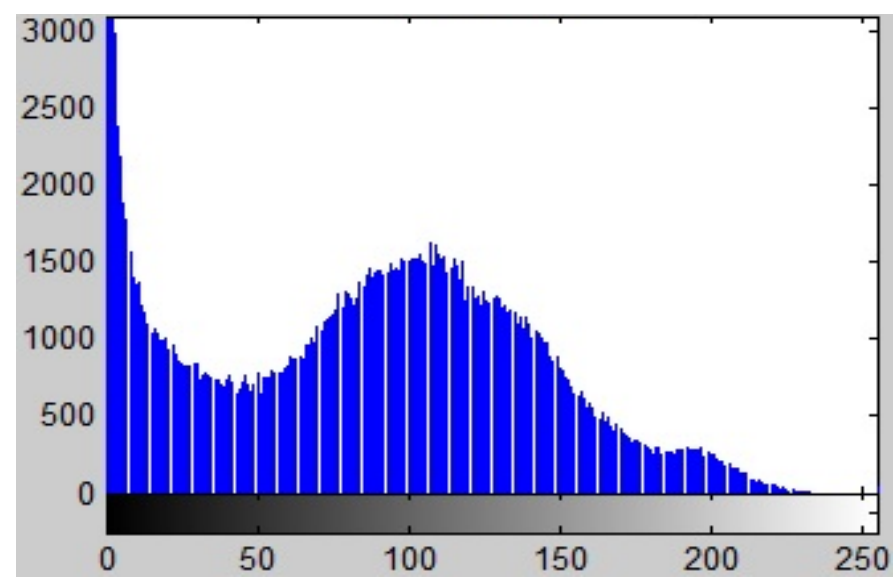

Fig 3. Histogram of Original MRI Prostate Image

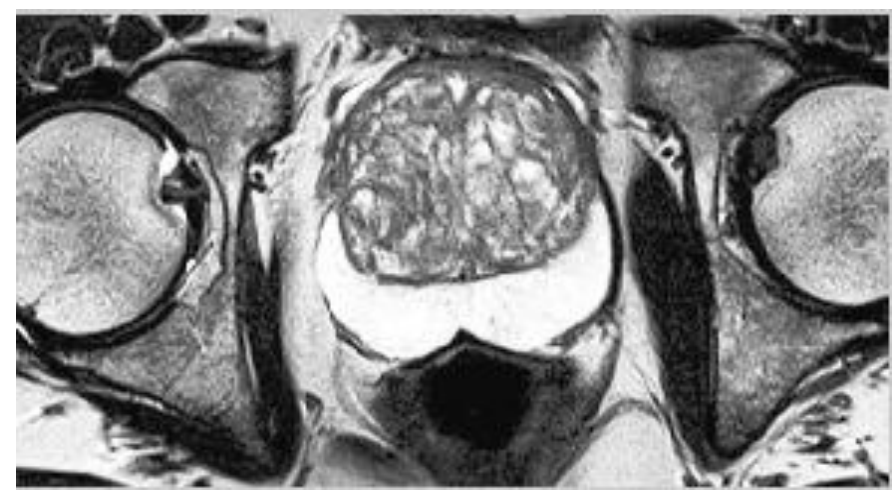

Fig 4. Histogram Equalized (HE) Image

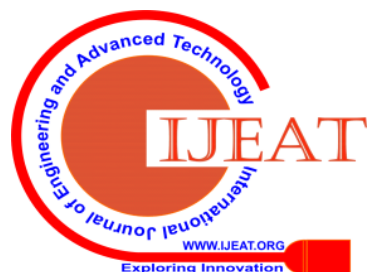




\section{Enhancement of MRI Prostate Image Using Histogram Equalization Techniques}

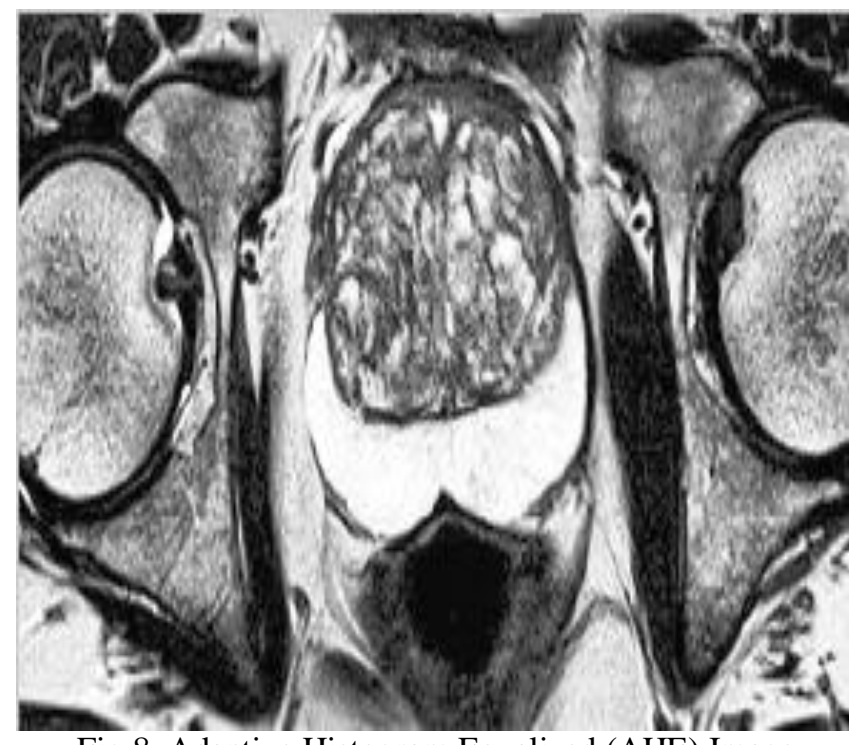

Fig 8. Adaptive Histogram Equalized (AHE) Image

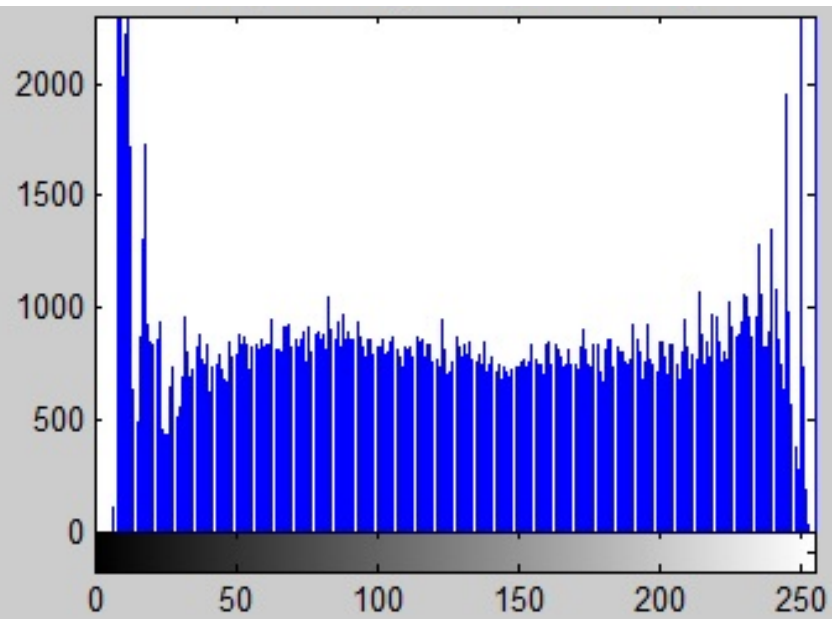

Fig 9. Histogram of Adaptive Histogram Equalized (AHE) Image

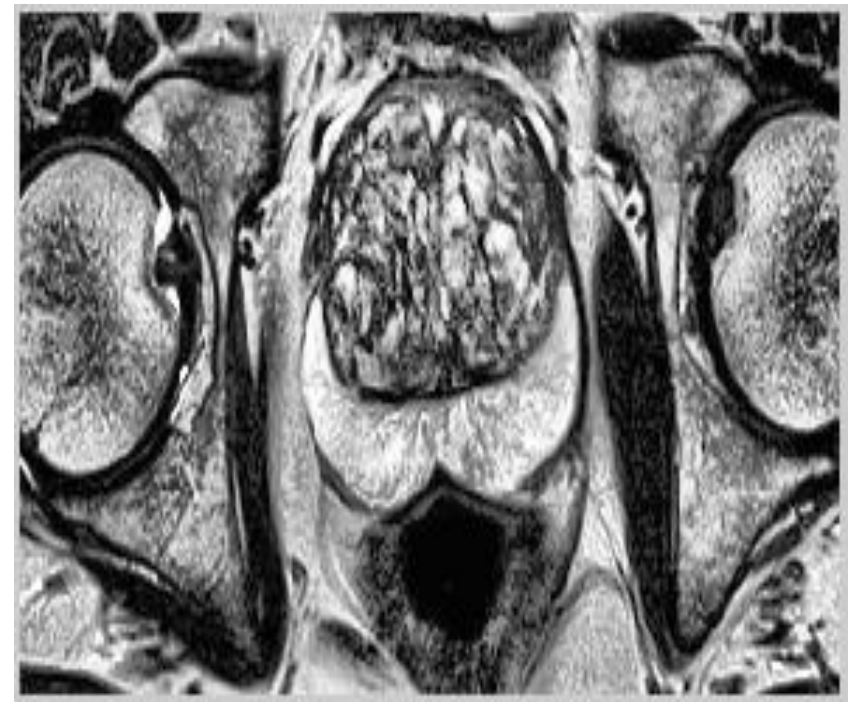

Fig 10. Contrast Limited Adaptive Histogram Equalized (CLAHE) Image

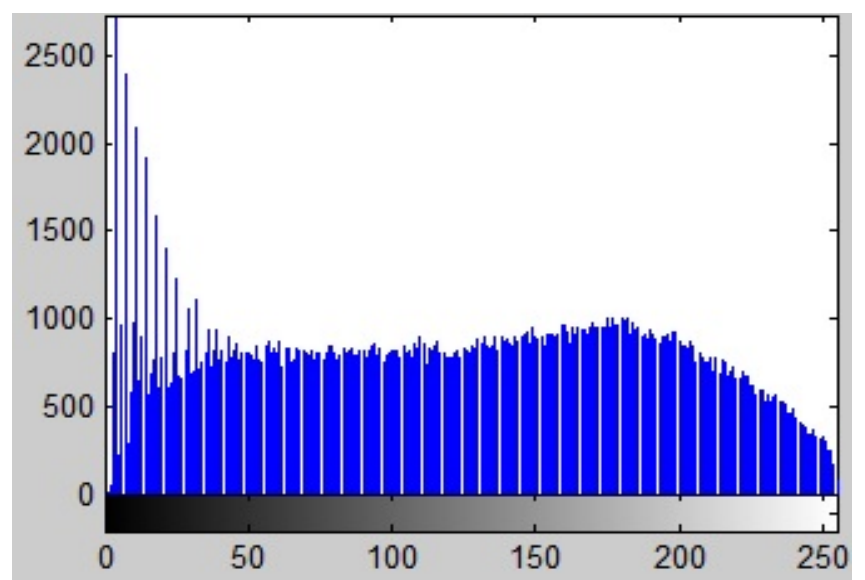

Fig 11. Histogram of Contrast Limited Adaptive Histogram Equalized (CLAHE) Image

Table 1. Shows the comparison of HE, LHE, AHE and CLAHE with the defined parameters.

\begin{tabular}{|l|r|r|r|r|}
\hline & HE & LHE & AHE & $\begin{array}{c}\text { CLAH } \\
\text { E }\end{array}$ \\
\hline Chi square & $3.29 \mathrm{E}+0$ & $3.40 \mathrm{E}+0$ & $3.65 \mathrm{E}+0$ & \\
& 3 & 3 & 3 & 0 \\
\hline Intersection & & & & \\
& 38741 & 55304 & 46374 & 262183 \\
\hline E-Canberra & & & $3.01 \mathrm{E}+0$ & \\
& 17.5974 & 21.8563 & 5 & 0 \\
\hline Euclidean, & $3.19 \mathrm{E}+0$ & $4.16 \mathrm{E}+0$ & $4.3176+0$ & \\
& 4 & 4 & 4 & 0 \\
\hline Dnon-IS, & 195324 & 208374 & 216842 & 0 \\
\hline $\begin{array}{l}\text { Square-Cho } \\
\text { rd }\end{array}$ & $2.87 \mathrm{E}+0$ & $3.01 \mathrm{E}+0$ & $3.13 \mathrm{E}+0$ & 5 \\
\hline
\end{tabular}

\section{CONCLUSION AND FUTURE SCOPE}

Histogram Equalization (HE) is more simple and an effective technique however it accurately changes the brightness of the image. Other methods like Local Histogram Equalization (LHE), Adaptive Histogram Equalization (AHE) and Contrast Limited Adaptive Histogram (CLAHE) are also useful to enhance the brightness of the image. When comparing to LHE and AHE, CLAHE is more time consuming technique. CLAHE is used to enhance the contrast of image and also it removes the noise yet at the same time tends to noise. In future work plans to introduce a new technique to enhance the MRI image more effectively with lesser time for execution when comparing to the already existing techniques.

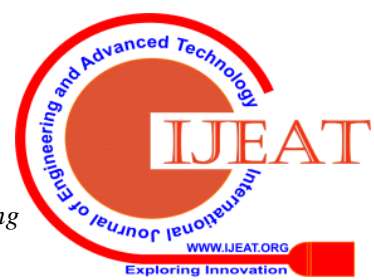




\section{REFERENCES:}

1. Sajid Ullah Khan, Najeeb Ullah, Imran Ahmed, Irshad Ahmad, Wang Yin Chai, "MRI Imaging, Comparison of MRI with other Modalities, Noise in MRI Images and Machine Learning Techniques for Noise Removal: A Review",Article in Current Medical Imaging Reviews, DOI: $\quad 10.2174 / 1573405614666180726124952$, July 2018

2. Hongmei Zhu, "Medical Image Processing Overview", https://pdfs.semanticscholar.org/4594/3c7f9170778ada 18fe0726fe9f90ff211dcc.pdf

3. Hardeep kaur, Jyoti Rani, "MRI brain image enhancement using Histogram equalization Techniques", IEEE WiSPNET 2016 conference, 978-1-4673-9338-6/16/\$31.00 @2016 IEEE

4. Sargun and Shashi B. Rana, "A Review of Medical Image Enhancement techniques for Image Processing", International Journal of Current Engineering and Technology, E-ISSN 2277 - 4106, P-ISSN 2347 - 5161, Vol.5, No.2,April 2015.

5. Sumaya, Zena Vatsa, "MRI Image Enhancement", International Journal of Science and Research (IJSR) ISSN (Online): 2319-7064, Volume 4 Issue 5, May 2015.

6. Ravindra Pal Singh and Manish Dixit, "Histogram Equalization: A Strong Technique for Image Enhancement", International Journal of Signal Processing, Image Processing and Pattern Recognition Vol.8, No.8 (2015), pp.345-352

7. Shefali Gupta, Yadwinder Kaur, " Review of Different Local and Global Contast Enhancment Techniques for Digital Image", International Journal of Computer Applications (0975 - 8887) Volume 100- No.18, August 2014

8. Julang Jiang, Yousheng Zhang, Feng Xue, Min Hu, "Local Histogram Equalization with Brightness Preservation", Acta Electronica Sinica, no. 5, pp. 861-866, 2006.

9. D. J. Ketchum. Real-time image enhancement techniques. Proc. SPIE/OSA, 74:120-125, 1976.

10. A. Rosenfeld and A. C. Kak. Digital Picture Processing. Computer Science and Applied Mathematics. Academic Press, 1982.

11. Rajesh Garg, Bhawna Mittal, Sheetal Garg, "Histogram Equalization Techniques For Image Enhancement", IJECT Vol. 2, Issue 1, March 2011

12.Brij Bhan Singh, Shailendra Patel, "Efficient Medical Image Enhancement using CLAHE Enhancement and Wavelet Fusion", International Journal of Computer Applications (0975 - 8887) Volume 167 - No.5, Jun

\section{AUTHORS PROFILE}

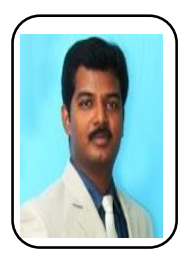

N.Gopinath , Assistant Professor, Department of Computer Science and Engineering, Sri Sairam Engineering College affiliated to Anna University, Chennai, (INDIA). Obtained Bachelor's Degree (B.Tech) in Information Technology from Anna University in 2009, Master's Degree (M.E) in Computer Science and Engineering from Anna University in 2011. Having 07+ years of teaching experience in Undergraduate level. Area of interests includes Image Processing, Data Mining and Networking. Published many papers in reputed journals and conferences. Member in IAENG.

Dr.A.Chinnasamy born on $26^{\text {th }}$ Nov 1981 in Salem district, Tamilnadu, India. He obtained his Bachelors degree (B.E) in Computer Science and Engineering from Anna University in 2005, Master degree (M.E) in Computer Science and Engineering from Anna University in 2008 and Ph.D., Information and Communication Engineering, Anna University, Chennai, 2017. He is currently working as Associate Professor in the Department of Computer science and Engineering at Sri Sairam Engineering College affiliated to Anna University, Chennai, (INDIA). He research interest is Wireless Communication. $\mathrm{He}$ is a life member of the Computer Society of India (CSI), ISTE, IAENG. Reviewer in Measurement and control Journal and Wireless Personal Communication

\begin{abstract}
M.SureshAnand, is working as an Associate professor/CSE in Sri Sairam engineering college, Chennai. He had published more than 50 papers in reputed journals and conferences. He is having 15 years of experience in teaching. His areas of interest are Image Processing, Computer Vision, Machine Learning, Human Computer Interaction and Deep Learning. He also member in IAENG \& IACSIT.
\end{abstract}


Enhancement of MRI Prostate Image Using Histogram Equalization Techniques

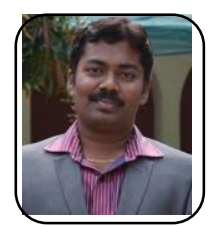

V. Pandimurugan, Assistant Professor in Hindustan University, Chennai. I did my B.E (EEE) in M.I.E.T Engg college, Trichy after that i finished my M.E(CSE) in M.N.M Jain Engg College, Chennai. I started my profession as ITO Trainee in Mphasis an EDS company, after that I joined in Hindustan University as Assistant Professor in the year of 2009. $6+y r s$ of Experience in Teaching Profession . 